\author{
Ketevan Margiani, Tamar Chankseliani \\ Ivane Javakhishvili Tbilisi State University, Tbilisi, Georgia
}

\title{
The Functional-Semantic Analysis of Particles Expressing Simple Negation in the Svan Language ${ }^{1}$
}

\begin{abstract}
In the Svan language there are more negative particles as compared to other Kartvelian languages - Georgian, Megrelian and Laz. The negative particles in the Svan language form three semantic groups expressing simple negation, negation of possibility and prohibition.

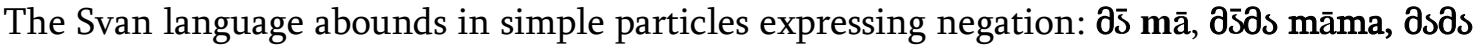

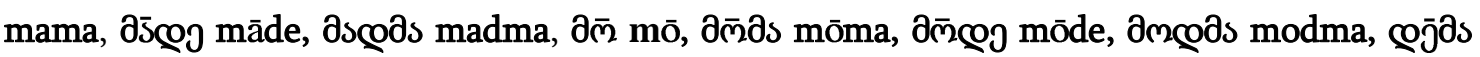

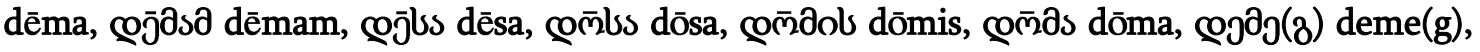
œว de, ৎəo dei...

These particles are considered as allomorphs. However, they can be differentiated based on functional and semantic criteria.

The functional differentiation has been carried out by T. Sharadzenidze who proved that the negative particles are used in different types of sentences based on the vowels $(\overline{\mathbf{a}} \overline{\mathrm{a}}, \mathbf{j} \mathbf{e}$ and $\mathrm{mo}$ ) which form part of these particles;

On the other hand, these particles are semantically diverse, as they express different degree of categoricity: some of them are neutral, non-categorical, and correspond to the Georgian sm/sms ar/ara (No/Not), while others reveal high degree of categoricity. Such particles are hard to translate. In the oral speech, their semantic nuances are revealed by means of intonation. This is natural, because categoricity and probability are semantically incompatible concepts. Their substitution with less categorical neutral negative particles yields a different interpretation of the subject's attitude to actions or events in the text.
\end{abstract}

Keywords: Svan language, negation, particle, semantics.

In the Svan language there are more negative particles as compared to other Kartvelian languages - Georgian, Megrelian and Laz. The abundance of negative particles in the Svan language is explained in the scientific literature as the result of dialectal variation. This fact must be verified. The negative particles in the Svan language form three semantic groups expressing simple negation, negation of possibility and prohibition. Neutral particles (expressing simple negation) are: $\partial \bar{s}$

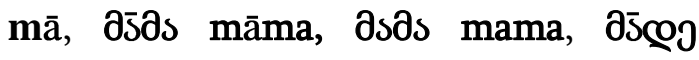

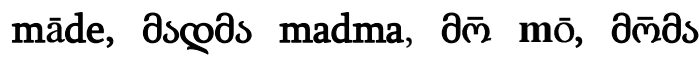

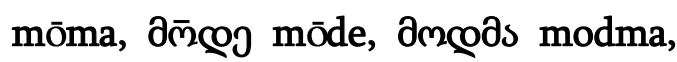

\footnotetext{
${ }^{1}$ The paper has been prepared within the scientific project - „The Category of Negation in the Kartvelian Languages"(\#FR17_388), implemented with the financial support of Shota Rustaveli National Science.
} 


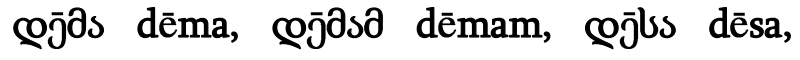

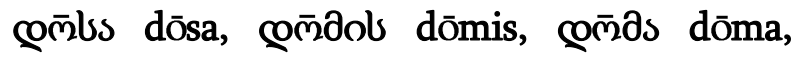

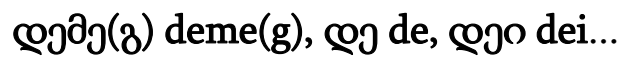

In the work "Negative Particles in the Svan Language" T. Sharadzenidze notes that "negative particles proper are: $\partial \bar{s} \_\partial \overline{\mathrm{m}}$

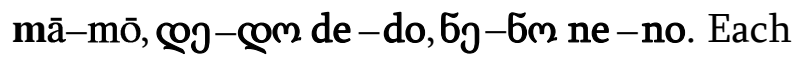
couple has the same consonantal element, whereas the vocalic element is different. Each member of the couple is combined with the same pronoun. Hence, we have

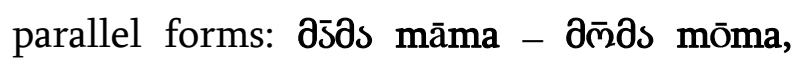

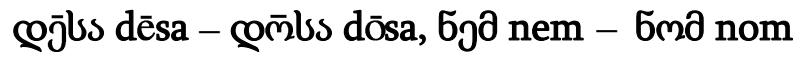
and so on" (Sharadzenidze 1946:315). Despite such diversity of particles under analysis, the scholar managed to carry out their functional differentiation and distinguished two groups of particles with different vowels. The scholar formulated a law according to which particles with different vowels are used in different sentences: "particles with vowels" $\bar{s} \bar{a}, \rho$ e "are used in the main indicative clauses of simple, compound and complex sentences and sentences with multiple parts if they do not contain the verb in the subjunctive mood. Particles with the vowel $m o$ are used in the subordinate clauses or simple sentences, sentences with multiple parts, compound and complex sentences which are not indicative and contain the verb in the subjunctive mood" (ibid, 325). This important law proves that the abundance of negative particles in the Svan language is not due to dialectal variations only, and it is possible to undertake their functional classification. T. Sharadzenidze also concludes that it is hard to identify every semantic nuance of each particle in this or that mood (Sharadzenidze 1946:289).

Our research has proved the truth of the above-mentioned statement. It has been observed that even the particles containing the same vowels cannot freely substitute

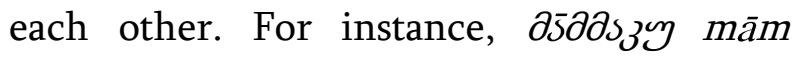

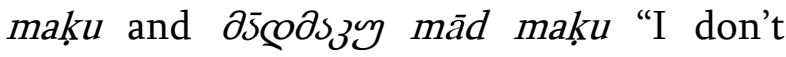
want" are stylistically and functionally different sintagmas in the Svan language. Even though, at first glance, both express simple neutral negation, they should be distinguished based on the degree of categoricity: the first construction denotes simple negation ("I don't want"), whereas the second construction is hard to translate: in this case, the subject categorically refuses to fulfill a certain action, i.e. the semantic 
nuance is a categorical attitude of the subject to a certain event:

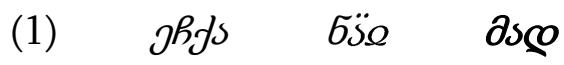
monzzふ̈бৎ̣ ečka näj mad otəgänd (The Svan Prose, volume I 1939:38,9) - "we didn't stop then".

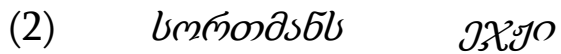

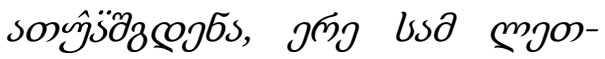

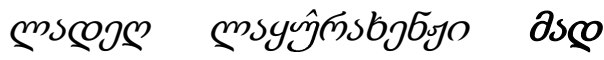

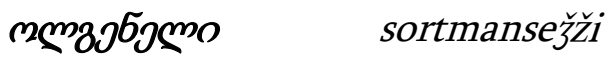

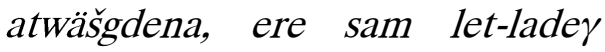
laqwraxenži mad olgeneli (The Svan Prose. Volume II 1957:13,25) - "Sortman was so offended that he did not rise from his bed for three days".

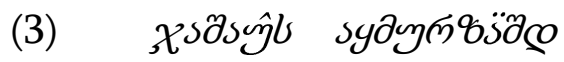

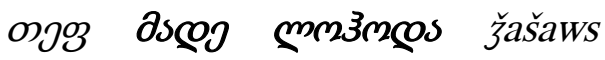
aqmurzäšd tep made lohoda (Readings in the Svan Language 1978:184,35) - "Jashav did not give the gun to Akmurza".

In the above contexts, the negation is obviously categorical. The use of particles

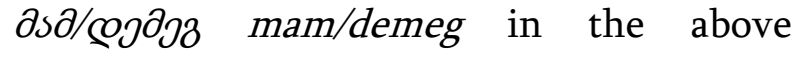
examples will not change the meaning of negation, but there will be less emphasis on categoricity, the intention of the subject who categorically refused to perform somebody else's will or implement a certain action upon someone's request.

The above-mentioned opinion is proved by another interesting fact: the particle $\partial \boldsymbol{m}^{\partial}$ moš, which, together with neutral negative particles, creates the meaning of potencialis and has the semantics of assumptionimpossibility, cannot be combined with the particles asco/mad, ascog/made, dscods/madma... These are the particles that denote categorical negation contextually.

It is quite clear why the particle $\partial m \partial \ddot{\partial} m o s ̌$ cannot be combined with the particles expressing categorical negation: categoricity and probability are incompatible concepts! This, in our opinion, is a sufficient argument proving that the negative particles of the Svan language should be divided based on their semantics of categoricity-neutrality (less categoricity).

Thus, the simple negative particles in the Svan language should be divided into two semantic sub-groups: 1) simple neutral particles denoting negation; 2) simple particles denoting categorical negation. The distribution of the lexemes under analysis into these groups, identification of certain 
laws based on their structure (for instance, repetition of consonants $\boldsymbol{\partial}$ and $\boldsymbol{\varrho}$ and their order in the structure of negative particles...) are the topics for further research. However, it can be assumed that the semantics of categoricity is obvious in those negative particles the structure of which has at least two consonants out of the three main clusters expressing negation identified by $\mathrm{T}$. Sharadzenidze (ds/ma, @o/de, 6m/no). The above-mentioned two consonantal clusters expressing categorical negation are as follows: $\partial s-c o$ ma-d, $\partial s-c o-\partial s m a-d-m a, \partial s-$

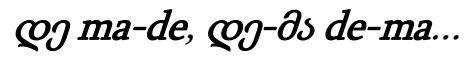

Conclusion: The Svan language abounds in simple particles expressing negation. These particles are considered as allomorphs. However, they can be differentiated based on functional and semantic criteria.

The functional differentiation has been carried out by T. Sharadzenidze who proved that the negative particles are used in different types of sentences based on the vowels ( $\bar{\jmath} \bar{a}, \jmath$, e and $\mathbf{m}$ o) which form part of these particles;

On the other hand, these particles are semantically diverse, as they express different degree of categoricity: some of them are neutral, non-categorical, and correspond to the Georgian sm/sms (No/Not), while others reveal high degree of categoricity. Such particles are hard to translate. In the oral speech, their semantic nuances are revealed by means of intonation. These particles cannot be combined with the particle $\partial \ddot{m}^{\prime}$ moš which has the meaning of potencialis. This is natural, because categoricity and probability are semantically incompatible concepts. Their substitution with less categorical neutral negative particles yields a different interpretation of the subject's attitude to actions or events in the text. 


\section{References}

T. Sharadzenidze. Negative Particles in the Svan Language. Iberian-Caucasian Linguistics, volume I. Publishing-House of the Georgian Academy of Sciences. Tbilisi. 1946.

Readings in the Svan Language. Tbilisi State University Publishing House. Tbilisi. 1978.

The Svan Prose. Volume I. Publishing-House of the Georgian Academy of Sciences. Tbilisi. 1939.

The Svan Prose.Volume II. Publishing-House of the Georgian Academy of Sciences. Tbilisi. 1957. 